Please do not remove this page

RMIT

UNIVERSITY

\title{
CNS+A capabilities for the integration of unmanned aircraft in controlled airspace
}

Gardi, Alessandro Giacomo Maria; Ramasamy, Subramanian; Sabatini, Roberto; Kistan, Trevor https://researchrepository.rmit.edu.au/esploro/outputs/9921859290401341/filesAndLinks?institution=61 RMIT_INST\&index=null

Gardi, A. G. M., Ramasamy, S., Sabatini, R., \& Kistan, T. (2016). CNS+A capabilities for the integration of unmanned aircraft in controlled airspace. Proceedings of the IEEE International Conference on Unmanned Aircraft Systems (ICUAS 2016), 779-788.

https://researchrepository.rmit.edu.au/discovery/fulldisplay/alma9921859290401341/61RMIT_INST:Resea rchRepository

Document Version: Accepted Manuscript

Repository homepage: https://researchrepository.rmit.edu.au

(C) 2016 IEEE

Downloaded On 2023/04/26 21:23:34 +1000

Please do not remove this page 
Thank you for downloading this document from the RMIT Research Repository.

The RMIT Research Repository is an open access database showcasing the research outputs of RMIT University researchers.

RMIT Research Repository: http://researchbank.rmit.edu.au/

\section{Citation:}

Gardi, A, Ramasamy, S, Sabatini, R and Kistan, T 2016, 'CNS+A capabilities for the integration of unmanned aircraft in controlled airspace', in Proceedings of the IEEE International Conference on Unmanned Aircraft Systems (ICUAS 2016), United States, 7-10 June 2016, pp. 779-788.

See this record in the RMIT Research Repository at:

https://researchbank.rmit.edu.au/view/rmit:37263

Version: Accepted Manuscript

Copyright Statement: (C) 2016 IEEE

Link to Published Version:

http://www.uasconferences.com/index.php 
This is the author pre-publication version. This paper does not include the changes arising from the revision, formatting and publishing process. The final paper that should be used for referencing is:

A. Gardi, S. Ramasamy, R. Sabatini and T.Kistan, "CNS+A Capabilities for the Integration of Unmanned Aircraft in Controlled Airspace." Proceedings of IEEE International Conference on Unmanned Aircraft Systems (ICUAS 2016). Arlington, VA (USA), June 2016.Print ISBN: 978-1-4673-9333-1

\section{CNS+A Capabilities for the Integration of Unmanned Aircraft in Controlled Airspace}

\author{
Alessandro Gardi, Subramanian Ramasamy \\ and Roberto Sabatini \\ RMIT University, Melbourne, Australia
}

\author{
Trevor Kistan \\ THALES Australia, Melbourne, Australia
}

\begin{abstract}
In this paper, the system requirements for the integration of Remotely Piloted Aircraft Systems (RPAS) in controlled airspace regions are discussed. The specificities in terms of Air Traffic Management (ATM) level of service, jurisdiction for deconfliction duties and prevalent traffic characteristics are analysed to support the identification of operational and equipage requirements for RPAS developers. Communication, Navigation, Surveillance, ATM and Avionics (CNS+A) equipment play an essential role in airspace regions characterised by high levels of Air Traffic Services (ATS) and a higher probability of traffic conflicts. A denser route structure and a more frequent occurrence of traffic conflicts mandate high CNS performance, as the deconfliction by ATM crucially relies on accurate and reliable CNS information. Notwithstanding, the reduced jurisdiction of aircraft in deconfliction duties also offers an opportunity to RPAS developers, as it relieves the requirements for on-board expert processing.
\end{abstract}

\section{INTRODUCTION}

Remotely Piloted Aerial Systems (RPAS) are at the forefront of current Research, Development, Test and Evaluation (RDT\&E) in both the civil and military aviation domains, and this is confirmed by the extraordinary growth in the number of operators and platforms. The quickly increasing adoption of RPAS poses well-known operational, technological and regulatory challenges, which shall be urgently addressed to ensure that the desired levels of safety and efficiency are retained for both manned and unmanned aircraft operations. Airspace segregation has been the most effective and hence widely adopted solution to enable RPAS operations, but in practice it bypasses the core problem of RPAS integration. This segregation meets widespread favour when it involves the layer closest to the ground and at the required distance from airports as the operation of manned aircraft in these regions is already denied. Yet this portion of airspace meets the operational requirements of very small RPAS only, and concerns are expressed when further extensions of airspace segregation are introduced at the expense of airspace available for manned aircraft.

In the United States, the National Aeronautics and Space Administration (NASA) is leading research and development efforts towards the introduction of systems specifically designed to manage Unmanned Aerial Systems (UAS) - the so-called UAS Traffic Management (UTM) system [1, 2]. As part of the NASA UTM program, commercial demands and proposals by major industrial operators are being reviewed in order to set the requirements and identify the optimal operational configuration [2-5]. NASA's UTM system is only slated for full implementation in controlled, non-segregated airspace in 2030 [6]. The need for interim solutions can be gauged from a recent study that identified 327 potentially hazardous encounters between RPAS and manned aircraft in the US during the period from December 2013 to September 2015, 90 of which involved commercial jets [7]. Prior to the introduction of specifically designed UTM systems, relatively simple modifications to current generation ATM systems would enable them to monitor RPAS and manage potential conflicts with other RPAS and with conventionally-piloted aircraft in nonsegregated controlled airspace. Lockheed Martin has already demonstrated the concept using the automated conflict probe of its Enroute Automation Modernization (ERAM) ATM system software [8].

\section{SHORT-TERM CHALlENGES}

A gradual integration of RPAS in the civil airspace starting from the less restrictive classes of airspace has been proposed and is traditionally favoured. Civil airspace is organised in up to seven classes from " $A$ " to " $G$ " in terms of decreasing level of Air Traffic Services (ATS) offered [9]. Correspondingly decreasing requirements in terms of Communication, Navigation, Surveillance (CNS) and Avionics (CNS+A) equipment, and in terms of compliance to Air Traffic Management (ATM) clearances are defined. Controlled airspace consists of control areas delineated so as to contain the flight paths of Instrument Flight Rules (IFR) flights or portions thereof for which it is desired to provide the applicable parts of the ATS [9]. Control areas also include ATS routes (airways) and Terminal Control Areas (TCA). Control zones and TCA complement control areas in their purpose and are specifically conceived to contain arrival and departure IFR flight paths in close proximity to the ground. For minor and isolated airports with limited IFR traffic, an appropriate combination of control zones and ATS routes is usually sufficient to safely 
This is the author pre-publication version. This paper does not include the changes arising from the revision, formatting and publishing process. The final paper that should be used for referencing is:

A. Gardi, S. Ramasamy, R. Sabatini and T.Kistan, "CNS+A Capabilities for the Integration of Unmanned Aircraft in Controlled Airspace." Proceedings of IEEE International Conference on Unmanned Aircraft Systems (ICUAS 2016). Arlington, VA (USA), June 2016.Print ISBN: 978-1-4673-9333-1

contain IFR traffic routes, as limited or no tactical deconfliction by ATM operators is required. Larger airports or combinations of multiple minor airports in close proximity to each other require more frequent tactical ATM interventions in terms of path stretching and separation measures. TCA are therefore established whenever additional allowance for these tactical measures needs to be introduced. In order to maximise safety of IFR traffic TCA are most commonly granted the highest levels of ATS, corresponding to airspace classes " $\mathrm{A}$ ", "B" or, less frequently, "C". The higher levels of ATS assigned to controlled airspace implicate that most of the responsibilities for conflict detection, separation maintenance, sequencing and spacing lie on the ATM side. This is effectively the opposite of recreational airspace assigned classes " $G$ " and " $F "$ and has important consequences for RPAS developers.

The less demanding regulatory requirements for equipment and operational obligations, especially in classes " $F$ " and "G", are certainly valued from the RPAS perspective, as their reliability is still lower than the one offered by manned aircraft [10]. Nevertheless, separation in these airspace regions is on pilots' responsibility and based on visual detection and deliberate execution of avoidance manoeuvres according to the rules of the air [11]. These considerations introduce substantially higher technological challenges for RPAS developers, as separation relies uniquely upon on board Detect And Avoid (DAA) systems and decisionmaking processes, hence higher levels of on board autonomous processing are required to mitigate the risks arising in connection to possible failures to the Command and Control (C2) loop involving the ground pilot.

Within controlled airspace, deconfliction duties are within the ATM jurisdiction and aircraft are expected to execute ATM directives after very limited review. The analysis of the ATM clearance by pilots is restricted to:

1. feasibility in terms of safe operational envelope;

2. suitability in terms of the short/medium term objectives of the mission;

In such an operational scenario, the algorithmic complexity necessary to grant RPAS the required autonomy is very limited, as RPAS are expected to execute ATM directives after very limited analysis. Conversely, in recreational airspace where deconfliction is responsibility of aircraft, substantially higher levels of expert processing are required at all times to grant the necessary autonomy [10]. Autonomy can be simply defined as the capability of making decisions without assistance by human operators and is essential in RPAS to both increase the utility of the platform and the safety of its operation as it allows the vehicle to avoid hazardous situations if failures occur in the C2 loop [12]. Levels of autonomy were initially defined by Sheridan [13]. Sheridan's 10-level scale of autonomy is based on the decision-maker (human or system) and on how the decisions are executed. The expert processing required to grant RPAS the appropriate levels of autonomy can be attained either by knowledge-based algorithms or by more advanced forms of machine intelligence. In both cases the algorithm size and complexity can grow considerably based on the number of factors and variables to be taken into account and on the inclusion of what-if scenario analysis processes. With respect to machine intelligence, the eventual integration of machine learning processes may also introduce a behaviour that is a priori non-deterministic, posing further challenges for certification authorities. One of the most pressing challenges associated with the development of the required trusted autonomy for unrestricted operations is the substantial complexity of flight critical software that needs to be integrated and reliably executed on-board [12]. In particular, researchers estimated that software size necessary to successfully integrate the autonomy required for unrestricted operations is well in excess of one million lines of code, which is an order of magnitude greater than manned aircraft equivalents [12]. Effective and certified failure management is also required to attain the desired level of safety for unrestricted operations. Nevertheless, the costs of certification could be managed by reducing the number of sub-systems and/or lines of code subject to certification requirements, as not all the autonomous decision-making in RPAS is for safety-critical purposes. Licensing already-certified software modules could also help manage these costs. However, unlike software safety, in terms of system safety requirements, new systems deploying "certified algorithms/software" still need to be certified themselves in very specific contexts, as the software may be running on different hardware, be configured differently, be employed in different operational contexts etc. One possible challenge for operation within controlled airspace is posed by groups or swarms of RPAS, which would easily saturate ATM capacity if considered as separate aircraft. In this perspective, regulations are already accommodating formation flying of manned aircraft [11], and formations of RPAS can be analogously considered as a single aircraft from the ATM perspective.

In summary, the high level of ATS in controlled airspace can be seen as an opportunity as it relieves RPAS developers from the requirement of integrating very high levels of autonomy, with the associated computational complexity. Nevertheless, the limited jurisdiction of aircraft in airspace classes characterised by high levels of ATS does not remove the requirements for RPAS to equip a certified DAA system, as visual detection and avoidance is also prescribed as a last resort in these classes of airspace.

\section{CNS+A REQUIREMENTS}

Comprehensively, in terms of granting the required levels of operational safety in airspace with high levels of ATS, the requirement is for $\mathrm{CNS}+\mathrm{A}$ equipment enabling the RPAS to reliably comply with ATM directives and be continuously 
This is the author pre-publication version. This paper does not include the changes arising from the revision, formatting and publishing process. The final paper that should be used for referencing is:

A. Gardi, S. Ramasamy, R. Sabatini and T.Kistan, "CNS+A Capabilities for the Integration of Unmanned Aircraft in Controlled Airspace." Proceedings of IEEE International Conference on Unmanned Aircraft Systems (ICUAS 2016). Arlington, VA (USA), June 2016.Print ISBN: 978-1-4673-9333-1

monitored in their execution, rather than integrating high levels of autonomy with the associated computational complexity. In this perspective, the fact that RPAS support appears as one of the key performance improvement areas identified by the ICAO for Aviation Systems Block Upgrade (ASBU) is certainly valuable [14]. As captured in the ASBU roadmap, the ongoing transition into TrajectoryBased Operations (TBO) paradigm is eliciting several important $\mathrm{CNS}+\mathrm{A}$ advancements including the development of 4DT algorithms for conflict-detection, planning, negotiation and validation that enable unrestricted access of RPAS to civil airspace. The following phases can therefore be outlined for the integration of RPAS in the 4D-TBO context:

- Initial integration of RPAS into non-segregated airspace: implementation of basic procedures and functions including DAA for operating RPAS.

- RPAS integration in traffic: implementation of defined procedures addressing lost link as well as enhanced DAA functions.

- RPAS transport management: implementation of RPAS operations on the airport surface and in nonsegregated airspace similar to manned aircraft.

The initial integration of RPAS requires capabilities including ground-based DAA systems and the adoption of a combination of policies, procedures, and technologies intended to facilitate safe airspace access. Cooperative and non-cooperative DAA performance-based requirements are currently being developed and have to be certified to support the RPAS operational improvements [14]. RPAS integration in traffic requires the development of an airborne DAA system, which must be able to fulfil the requirements for MAC avoidance in non-segregated airspace for both cooperative and non-cooperative targets [15]. In particular, this technology enabler will cope with air and ground obstacles of various characteristics (natural and man-made) including long and thin structures such as electrical cables, poles and aerial obstacles such as other RPAS and manned aircraft [16].

In order to progress towards a full integration of RPAS into the non-segregated airspace the integration of the following elements is required:

- Beyond Line-of-Sight (BLoS) communication systems.

- High-integrity airborne and ground-based RPAS navigation systems and integrated failsafe avionics architectures.

- Fused cooperative/non-cooperative surveillance systems incorporating collision avoidance and collaborative conflict resolution capabilities in a network centric operational scenario.

- Automation functions and standards for Human-InThe-Loop (HITL) interactions
The Joint Architecture for Unmanned Systems (JAUS) standard Domain Model (DM) and Reference Architecture (RA) provide mechanisms for RPAS interoperability including integration into the airspace, architecture framework, message formats and a set of standard messages [17]. In the long term, RPAS will not require on board speech recognition as ATM instructions will consist exclusively of digitally codified 4DT directives. In the medium-term, RPAS may however implement simple speech synthesis in order to respond to ATM instructions, as the maturity attained by this technology is adequate. For instance, successful experimental flight trials have already been reported with speech recognition on the ATM side converting spoken controller instructions into datalinkmediated directives $[18,19]$.

\section{RPAS FLIGHT MANAGEMENT SYSTEMS}

Flight Management Systems (FMS) are the avionics equipment primarily responsible for mission planning, automated guidance and navigation services. In particular, the Guidance, Navigation and Control (GNC) functionalities integrated in the FMS are:

- Lateral and vertical navigation (state determination, position estimation, navigation radio tuning, data fusion and polar navigation).

- Trajectory computation, estimation and vertical speed/altitude profile optimisation.

- Lateral and vertical guidance (information for autopilot and flight director).

- Performance predictions.

- Determination of input commands for the AutoThrottle (A/THR) system.

- Continuous monitoring and correction of the flight path.

On board manned aircraft, the FMS also integrates suitable pilot and co-pilot interfaces. Next Generation Flight Management Systems (NG-FMS) are FMS that fulfil the operational, safety and environmental requirements to deploy the 4D-TBO capabilities. The key functionalities of NG-FMS for TBO are:

- Multi-Objective 4D Trajectory Optimisation (MOTO4D) for both flight planning and real-time ATM transactions.

- 4D trajectory negotiation/validation with a groundbased ATM 4DT Planning, Negotiation and Validation (4-PNV) Decision Support System (DSS).

- Real-time information exchange, processing and updating.

Each aircraft equipped with NG-FMS generates 4DT intents, defined according to the Flight Management Computer (FMC) ARINC 702A-3 characteristic as a string 
This is the author pre-publication version. This paper does not include the changes arising from the revision, formatting and publishing process. The final paper that should be used for referencing is:

A. Gardi, S. Ramasamy, R. Sabatini and T.Kistan, "CNS+A Capabilities for the Integration of Unmanned Aircraft in Controlled Airspace." Proceedings of IEEE International Conference on Unmanned Aircraft Systems (ICUAS 2016). Arlington, VA (USA), June 2016.Print ISBN: 978-1-4673-9333-1

of 4DT points that define the predicted trajectory of the aircraft along with the point type and turn radius associated with the flight path transition [20]. Intent data are updated in situations of a change in the nominal flight path, addition of new sequencing points and weather data. The 4DT computation and optimisation component of the NG-FMS is reconfigurable with that of the ground-based counterpart to enable negotiation and validation updates in real-time. Additionally, the intents are recomputed according to flight plan revisions, weather updates, guidance mode modification, cost index modification and corrections for position uncertainties in real-time based on the 4DT optimisation algorithms. Subject to various in-flight changes, trajectory calculations are refreshed to maintain consistency and downlinked to the 4-PNV via the NG-ADL. FMS for RPAS not only integrate the ensemble of functionalities provided by FMS of manned aircraft, but also include a number of functionalities specifically designed to compensate for the lack of an on-board pilot. In this perspective, the RPAS FMS function is to reduce the ground control pilot's workload by acting both as a mission planner and a mission monitor. Additionally, due to the growing public concerns, research is currently being carried out to specifically improve operational efficiency and mitigate environmental impacts. NG-FMS for RPAS are currently being developed for 4DT-TBO in combination with groundbased 4-PNV systems and NG-ADL.

Comprehensively, the CNS+A systems for RPAS are illustrated in Figure 1, where airborne systems (coloured) and subsystems (white) are represented by rectangular blocks, while ground-based systems are represented by hexagonal blocks.

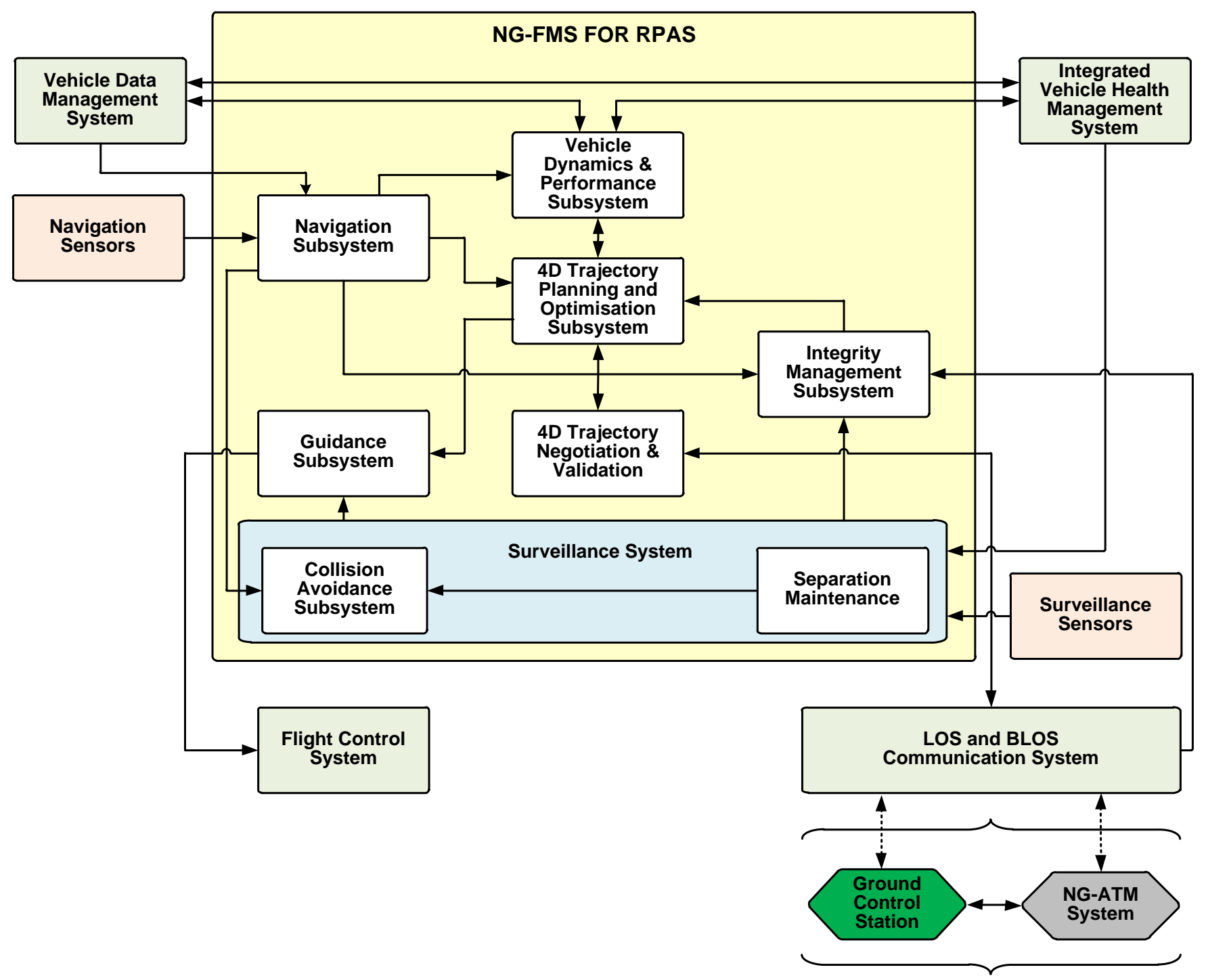

Fig. 1. CNS+A systems for RPAS. 
This is the author pre-publication version. This paper does not include the changes arising from the revision, formatting and publishing process. The final paper that should be used for referencing is:

A. Gardi, S. Ramasamy, R. Sabatini and T.Kistan, "CNS+A Capabilities for the Integration of Unmanned Aircraft in Controlled Airspace." Proceedings of IEEE International Conference on Unmanned Aircraft Systems (ICUAS 2016). Arlington, VA (USA), June 2016.Print ISBN: 978-1-4673-9333-1

The core NG-FMS modules are:

1. Trajectory Planning/Optimisation - This module performs 4DT planning for pre-tactical, tactical and emergency situations. To maximise operational efficiency of the planned trajectory, the trajectory planning module is based on Multi-Objective 4D Trajectory Optimisation (MOTO-4D) algorithms. The MOTO-4D suite includes various models, databases, and operational/economic/environmental optimality criteria and allows an arbitrary number of constraints to be introduced. The integrated databases include navigation, performance, magnetic deviation and environmental databases. Pre-defined cost functions include minimisation of fuel consumption, flight time, operative costs, noise impacts, emissions and contrails.

2. Trajectory Monitoring - It performs state estimation, calculating the deviations between the active 4DT intents and the estimated/predicted aircraft states.

3. Path Correction - It corrects the path deviation in terms of lateral, vertical and time profiles and the generated steering commands are provided to the guidance module of the NG-FMS.

4. Trajectory Negotiation and Validation - It carries out the process of negotiation that can be initiated by the pilot via the NG-FMS, making use of the information available on board, or by the NG-ATM system, supervised by the Air Traffic Control Operator (ATCO).

5. Performance Manager - It monitors the active 4DT intents for potential performance violations, to address integrity requirements. The integrity analysis module is based on RNP, RCP and RSP managers.

Integrity Manager - This module is used to generate integrity caution (predictive) and warning (reactive) flags based on inputs from different sensors/systems and predefined decision logics. A loss of data leads to reinitialising of the trajectory planning and subsequently the 4DT generation and optimisation process. For instance, the main causes of GNSS signal outage and degradation in flight, namely: antenna obscuration, multipath, fading due to adverse geometry and Doppler shift are identified and modelled to implement integrity thresholds and guidance algorithms in the Avionics-Based Integrity Augmentation (ABIA) system [21-24].

\section{OPTIMAL 4D TRAJECTORY PLANNING}

Automatic 4DT generation, negotiation and validation functionalities supporting deconfliction, rerouting and rescheduling in real-time are essential elements of the $\mathrm{CNS}+\mathrm{A}$ enabled concept of operation introduced in [25]. Real-time air-ground transactions ensure the validated 4DT are updated frequently and forcibly when any change in operational conditions emerges. As mentioned above, the automated 4DT planning component of $\mathrm{CNS}+\mathrm{A}$ system implements suitable MOTO algorithms. A review of various MOTO techniques proposed in the literature for the optimisation of aircraft flight trajectories is given in [26]. The capability of NG-FMS to generate optimal 4DT exploiting suitably implemented MOTO algorithms was investigated in $[27,28]$. The real-time MOTO-4D algorithms implemented in the NG-FMS adopt the same models implemented in the 4-PNV. The aircraft dynamic parameters are shared between the NG-FMS and the 4-PNV through the NG-ADL, along with the relevant aircraft states, to ensure synchronization and mathematical consistency. The adopted three degrees of freedom (3DOF) point-mass dynamics model with variable mass is:

$$
\dot{\boldsymbol{x}}=\left\{\begin{array}{l}
\dot{v}=\frac{\tau \cdot T_{C L}-D}{m}-g \sin \gamma \\
\dot{\gamma}=\frac{g}{v} \cdot(N \cos \mu-\cos \gamma) \\
\dot{\chi}=\frac{g}{v} \cdot \frac{N \sin \mu}{\cos \gamma} \\
\dot{\phi}=\frac{v \cos \gamma \sin \chi+v_{w_{\phi}}}{R_{E}+z} \\
\dot{\lambda}=\frac{v \cos \gamma \cos \chi+v_{w_{\lambda}}}{\left(R_{E}+z\right) \cos \phi} \\
\dot{z}=v \sin \gamma+v_{w_{z}} \\
\dot{m}=-F F
\end{array}\right.
$$

Where $v$ is the true airspeed, $\boldsymbol{v}_{\boldsymbol{w}}$ is the wind velocity vector, $\gamma$ is the flight path angle, $\chi$ is the track angle, $m$ is the aircraft mass, $\phi \lambda$ and $z$ are respectively the geodetic latitude, longitude and altitude, $g$ is the gravity acceleration, $R_{E}$ is the geodetic Earth radius, $D$ is the aircraft drag, $T_{C L}$ is the maximum climb thrust. The control variables are $\boldsymbol{u}=\{N, \tau, \mu\}$, which respectively represent the load factor, the throttle and the bank angle. The drag is calculated with the conventional parabolic approximation as:

$$
D=\frac{1}{2} \rho v^{2} S C_{D 0}+\frac{2 C_{D 2} m^{2} g^{2}}{\rho v^{2} S}
$$

Where $\rho$ is the local air density, and $S, C_{D 0}, C_{D 2}$ are respectively the aerodynamic reference surface and the two parabolic drag coefficients. The NG-FMS receives the controlled time of arrival target defined by the 4-PNV DSS, which becomes the Required Time of Arrival (RTA) to be used by the NG-FMS in determining the optimal trajectory states (final time). The Estimated Time of Arrival (ETA) may be computed at multiple fixes along the flight path. In general, a specific performance objective can be defined for each route segment. This performance objective is a multiobjective generalisation of the Cost Index (CI) that allowed an optimal selection of Calibrated Air Speed (CAS) / Mach number based on time and fuel costs only. The cost index allows the mission operators to weigh time, fuel, emissions, noise and other costs. The time cost, $J_{\text {time }}$ is given by: 
This is the author pre-publication version. This paper does not include the changes arising from the revision, formatting and publishing process. The final paper that should be used for referencing is:

A. Gardi, S. Ramasamy, R. Sabatini and T.Kistan, "CNS+A Capabilities for the Integration of Unmanned Aircraft in Controlled Airspace." Proceedings of IEEE International Conference on Unmanned Aircraft Systems (ICUAS 2016). Arlington, VA (USA), June 2016.Print ISBN: 978-1-4673-9333-1

$$
J_{\text {time }}=K_{t} t_{f}
$$

For RPAS adopting internal combustion engines, fuel consumption optimisation is achieved by implementing a Fuel Flow (FF) model. In terms of emissions, although engine design and other factors may influence total amounts, pollutant emissions are considered as a function of fuel flow, multiplied by specific emission factors, $\varrho$. Hence, the mathematical description of the emission performance index, $J_{\text {emission }}$, defined with respect to emissions, $e$ and time, $t$ is given by:

$$
J_{e}=\int_{t_{0}}^{t_{f}} \frac{d e}{d t}=\left(m\left(t_{f}\right)-m\left(t_{0}\right)\right) \varrho
$$

Each performance index provides a quantitative measure of the attainment of a specific objective and different objectives are typically conflicting, and thus the optimisation in terms of two or more objectives leads to a number of possible compromise choices, which are still optimal. Therefore, it is necessary to adopt suitable trade-off decision logics. In the aviation domain, single and biobjective optimisation techniques have been exploited for decades but they accounted only for flight time-related costs and fuel-related costs. These techniques have also been implemented in a number of current generation FMS in terms of the $C I$, which is a scalar value to balance the relative weighting of fuel and time costs. In the NG-FMS, the weightings are varied dynamically among the different flight phases of the flight.

Since computational times are a crucial aspect in the 4DT planning algorithm development, an a priori articulation of preference involving the weighted sum of the various performance indexes $J_{i}$ is employed to combine the multiple conflicting operational, economic and environmental objectives. The numerical algorithm for the solution of the optimal control problem with respect of the resulting combined objective is represented in Fig. 2. The currently employed 4DT optimization algorithm is based on direct solution methods of the global orthogonal collocation category, which are arguably the most effective solution methods currently available in terms of computational speed for the iterative solution of nonlinear optimization problems [29]. Additionally, to further enhance the algorithm stability and convergence performances, path constraints and boundary conditions are automatically strengthened on all state and control variables to restrict the search domain as much as feasible.

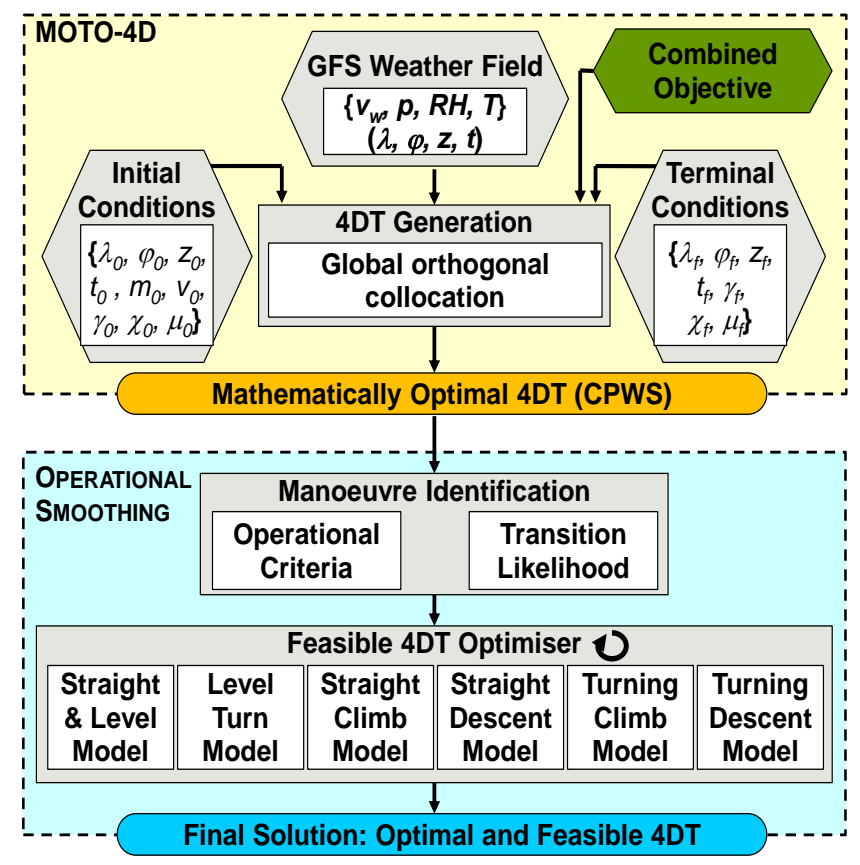

Fig. 2. Block diagram of the 4DT optimisation algorithm.

In the implemented MOTO-4D algorithm, a mathematically optimal 4DT is generated by the numerical solution algorithm. This 4DT is a discretised version of a Continuous/Piecewise Smooth (CPWS) curve, which in general may not be flyable by human pilots nor by conventional Automatic Flight Control Systems (AFCS), as it includes transition manoeuvres involving multiple simultaneous variations in the control inputs. Moreover, the discretised CPWS often consists of a very high number of 4D waypoints, which would have unacceptable impacts on the NG-ADL bandwidth. Therefore, a post-processing stage is introduced to segment the trajectory in feasible flight legs, including straight and level flight, straight climbs and descents, level turns, and climbing/descending turns. The final result is a concisely described 4DT consisting of feasible flight segments. The 4DT intent data include 4D waypoints (latitude, longitude, altitude and time), fly-by/flyover turn parameters, as well as performance criteria and restrictions. The lateral path is constructed in terms of segments (straight and turns), whose geometric characteristics depend on the required course change $\delta \chi$ and the predicted Ground Speed (GS) of the aircraft during turns. The NG-FMS computes turn radius $R$ and True Air Speed $v_{\text {TAS }}$ based on the selected altitude and takes into account the predicted wind speed $v_{\text {wind }}$ at that altitude. The bank angle $\mu$ is selected based on the RPAS dynamics, altitude and ATM restrictions. The radius $R$ of a turn can be conservatively calculated based on the maximum GS of the RPAS during the turn as:

$$
R=\frac{G S^{2}}{g \cdot \tan \mu}
$$


This is the author pre-publication version. This paper does not include the changes arising from the revision, formatting and publishing process. The final paper that should be used for referencing is:

A. Gardi, S. Ramasamy, R. Sabatini and T.Kistan, "CNS+A Capabilities for the Integration of Unmanned Aircraft in Controlled Airspace." Proceedings of IEEE International Conference on Unmanned Aircraft Systems (ICUAS 2016). Arlington, VA (USA), June 2016.Print ISBN: 978-1-4673-9333-1

where $g$ is the gravity acceleration module. The turn arc length $\Lambda$ is simply given by:

$$
\Lambda=\delta \chi \cdot R
$$

where the track change $\delta \chi$ is the difference in radians between the final and the initial ground tracks. The following prescriptions on $\delta \chi, R$, lead distance $Y$ (from the turn initiation to the 4DT waypoint) and abeam distance $X$ (between the 4DT waypoint and the point of the circular arc abeam it) are implemented to plan fixed-radius fly-by turns that comply with RTCA DO-229D, DO-236C and DO-283B [30, 31]:

1. The fly-by transition is defined by equation combined with the following equations [30]:

$$
\begin{gathered}
Y=\mathrm{R} \tan (0.5 \delta \chi) \\
\mathrm{GS}=\left|\overrightarrow{\mathrm{v}}_{\mathrm{TAS}}+\overrightarrow{\mathrm{v}}_{\mathrm{wind}}\right|=\left\{\begin{array}{l}
500 \text { kts } \text { (low altitude) } \\
750 \text { kts } \text { (high altitude) }
\end{array}\right. \\
\mu=\left\{\begin{array}{r}
\min \left[0.5 \delta \chi ; 23.0^{\circ}\right] \text { (low altitude) } \\
5.0^{\circ}
\end{array}\right. \\
\text { (high altitude) }
\end{gathered}
$$

2. The geometry of the Fixed Radius Transition (FRT) is defined by the track change $\delta \chi$ and the radius $\mathrm{R}$. The Lead Distance $Y$ and the Abeam Distance $X$ are defined based on the radius and the track change as per the following equations:

$$
\begin{gathered}
X=R \cdot\left(\frac{1}{\cos \delta \chi / 2}-1\right) \\
Y=R \cdot \tan (\delta \chi / 2)
\end{gathered}
$$

3. When transitioning from one airway to another, if both require a FRT at the common waypoint, the smaller of the two radii applicable shall be selected. In the case one of the two airways does not involve a FRT, the FRT of the other shall be implemented.

The criteria for implementing the definitions above in different flight phases and at various altitudes are given in Table 2-7 of RTCA DO-229D [31].

The errors in aircraft positioning are conveniently described by a navigation error ellipsoid. For cooperative and noncooperative obstacle avoidance and safe-separation maintenance, the overall uncertainty volume is obtained by combining the navigation error ellipsoid with the tracking error ellipsoid and then translating them to unified range and bearing uncertainty descriptors.

\section{NEGOTIATION AND VALIDATION}

In order to support deconfliction and rerouting operations performed at strategic and tactical online timeframes, strict time restrictions were set for the negotiation and validation processes. In particular, in line with the timeframe definition introduced in [32], the entire process of constraint calculation, 4DT generation, negotiation and validation including suitable time for review by human operators should be accomplished in less than the minimum time limit of the tactical online operation, corresponding to 180 seconds. To prevent endless loops that may easily violate the requirement, the development targeted single-attempt negotiation. EUROCONTROL's DOC 97-70-14 [33] was used as a guideline for implementing the negotiation loops, which were originally presented in [34] and improved in [35]. The updated negotiation loops are depicted in Fig. 3 and Fig. 4, which respectively represent the 4-PNV initiated and the NG-FMS initiated loops. The shared 4DT intents include the aircraft's unique identification and model, the wake-turbulence category, and the vector of 4DT segments. The 4-PNV system is the protagonist of the strategic online scenario as it retains a continuously updated global situational awareness. Unpredicted events prompt the 4PNV to initiate a strategic replanning and negotiation by uplinking new constraints to the NG-FMS, triggering onboard 4DT optimization. Alternatively, the 4-PNV may compute optimal 4DT and uplink them for validation by the aircraft. If, after on-board validation, constraint violations are detected (e.g. turn radius, climb rate), the aircraft downlinks a rejection message together with a new intent.

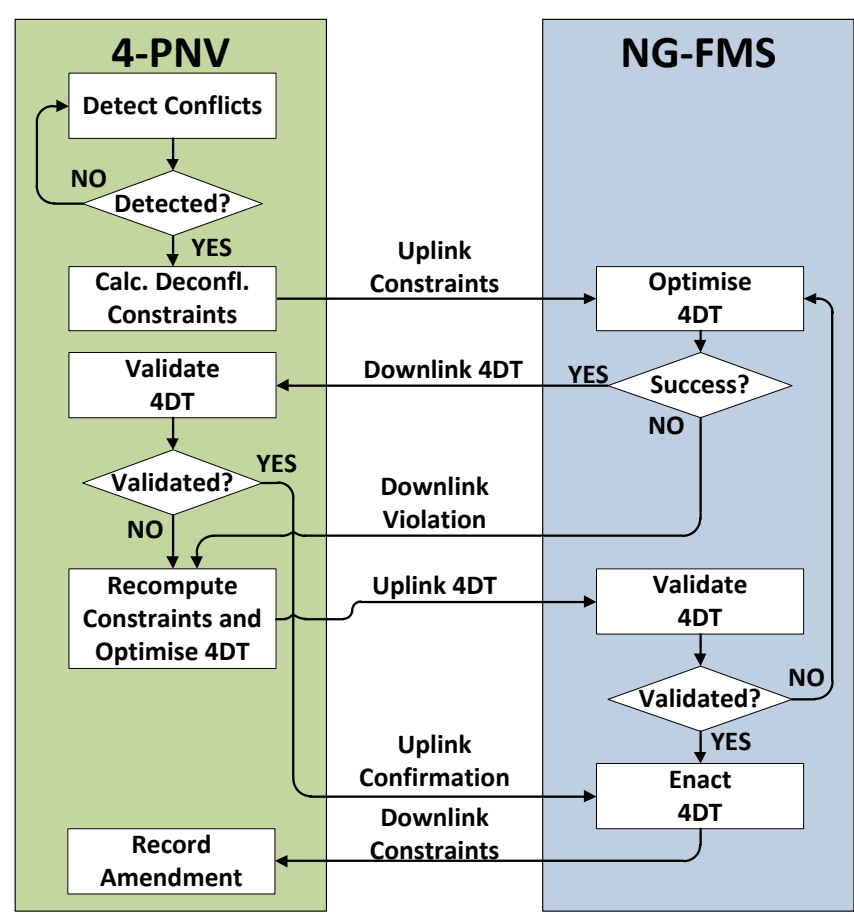

Fig. 3. 4-PNV initiated 4DT Intent Negotiation/Validation Loop. 
This is the author pre-publication version. This paper does not include the changes arising from the revision, formatting and publishing process. The final paper that should be used for referencing is:

A. Gardi, S. Ramasamy, R. Sabatini and T.Kistan, "CNS+A Capabilities for the Integration of Unmanned Aircraft in Controlled Airspace." Proceedings of IEEE International Conference on Unmanned Aircraft Systems (ICUAS 2016). Arlington, VA (USA), June 2016.Print ISBN: 978-1-4673-9333-1

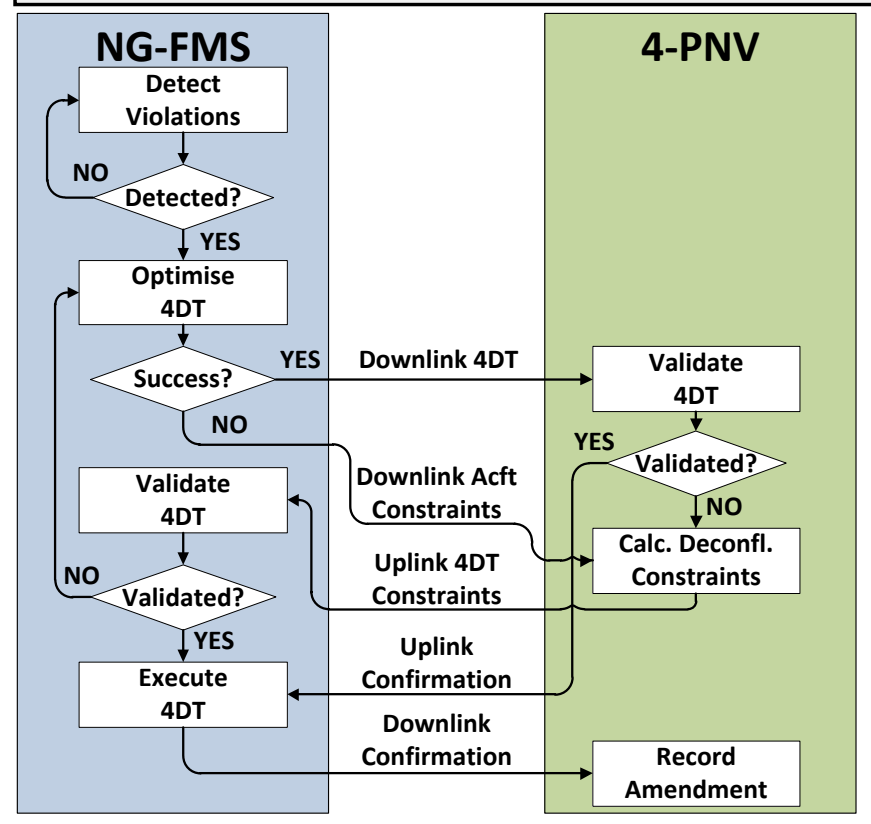

Fig. 4. NG-FMS initiated 4DT Intent Negotiation/Validation Loop

Multiple negotiation loops are allowed in the strategic online scenario but minimized thanks to the availability of multiple intents for each aircraft. In the tactical online scenario, either the NG-FMS or the 4-PNV may initiate intent negotiations. The 4-PNV will act mainly as a key decision maker. The NG-FMS may initiate the negotiation due to locally detected weather changes, aircraft performance degradation, equipment failures or on-board emergency situations. Other manoeuvre-related factors such as inefficient heading changes, and unachievable climb/descent rates and altitudes due to the actual aircraft weight may also be causes of negotiation. In the tactical online scenario, a "single-loop" negotiation is ultimately sought due to the reduced time and stringent traffic management commitments. 4DT are checked for traffic conflicts and separation from hazardous phenomena. The validation algorithm assesses the lateral and vertical separation criteria and includes a simplified wake vortex modelling to assess the longitudinal separation.

\section{Simulation CASE StUdy}

The simulation case study presented here investigates the operational integration of RPAS traffic with manned traffic within the controlled airspace of a medium-size airport. The traffic has to be sequenced and spaced before the shared final approach leg. This can be translated in 4DT constraints on selected waypoints. This capability, allows ATM operators to merge the RPAS with conventional traffic at any arbitrary merge point, ensuring the required separation minima are maintained at all times. The simulated AEROSONDE RPAS variant departs St Leonards Airfield (YSLE, S38.166 E144.687), and descends into Point Cook Royal Australian Air Force Base (YMPC, S37.933,
E144.753) after operating within the AEROSONDE Test Range (S38.210 E144.860), at altitudes between 1000 and $1500 \mathrm{ft}$, encompassed by Danger Area YMMM/D322A of the Melbourne Flight Information Region (FIR). Objectives such as fuel consumption, time and emissions are introduced in the trajectory optimization problem based on the information exchanged as a result of the 4DT negotiation process. The climb trajectory is depicted in Fig. 5, while the descent trajectory is depicted in Fig. 6.

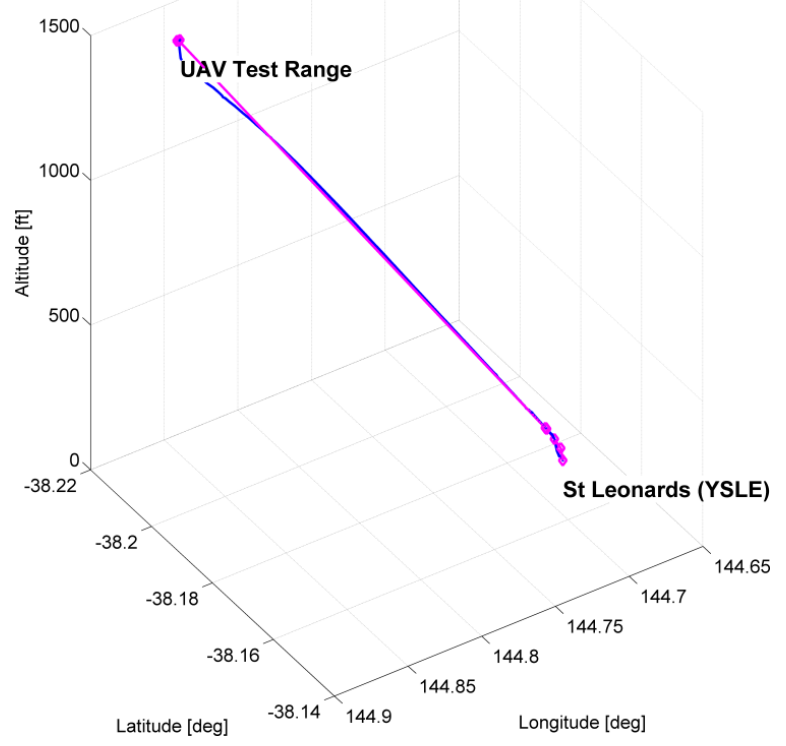

Fig. 5. Generated 4DT for the climb phase [27].

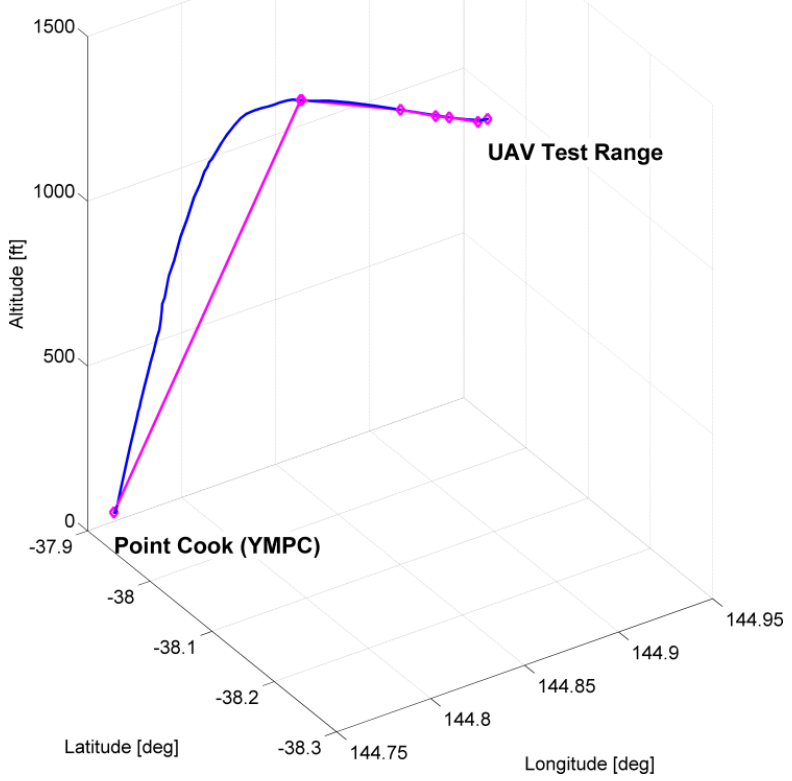

Fig. 6. Generated 4DT for the descent phase [27]. 
This is the author pre-publication version. This paper does not include the changes arising from the revision, formatting and publishing process. The final paper that should be used for referencing is:

A. Gardi, S. Ramasamy, R. Sabatini and T.Kistan, "CNS+A Capabilities for the Integration of Unmanned Aircraft in Controlled Airspace." Proceedings of IEEE International Conference on Unmanned Aircraft Systems (ICUAS 2016). Arlington, VA (USA), June 2016.Print ISBN: 978-1-4673-9333-1

The operational smoothing algorithm, described in [35] was adopted, and the resulting simplified and flyable trajectory is depicted in magenta. The NG-FMS generated the optimal climb and descent trajectories in 47 seconds. The trajectory corresponding to minimum fuel burn is characterized by a fuel saving of $130 \mathrm{~g}$ when compared to the minimum time case. A stochastic analysis case study was performed to evaluate the potential impact of system uncertainties on the trajectory generation process. The introduction of uncertainties on all nominal parameters, with ranges equal to the standard deviations allows transforming the EoM into stochastic differential equations that are then treated with the Monte Carlo sampling technique and solved using the deterministic optimizer for 100 samples [36]. In these simulations, real weather data obtained with the Global Forecast System (GFS) kindly made available by the National Climatic Data Center (NCDC) of the US National Oceanic and Atmospheric Administration (NOAA) was adopted, as described in [35].

\section{CONCLUSIONS}

In this paper we discussed the opportunities and challenges associated with the operation of Remotely Piloted Aircraft Systems (RPAS) in controlled airspace. The specificities of this domain are analysed in detail to extract the key factors affecting RPAS in terms of their equipment and operation. Challenges, in particular, are associated with the separation maintenance from conventional aircraft traffic, especially in the presence of frequently intersecting flight paths of departures, arrivals and overflights, resulting in a need for frequent tactical interventions by Air Traffic Management (ATM) operators. Conversely, some advantages are envisaged in relation to the very high level of jurisdiction on the ATM side, which diminishes the requirements for expert processing capabilities on board the RPAS. Additionally, capacity benefits associated with lower longitudinal separations are mentioned. Based on the analysis, requirements for unrestricted operation of RPAS in controlled airspace are translated in Communication, Navigation, Surveillance, ATM (CNS/ATM) and Avionics $(\mathrm{CNS}+\mathrm{A})$ equipment. The paper therefore describes the current state-of-the-art and the research activities in progress to implement the required $\mathrm{CNS}+\mathrm{A}$ capabilities on board RPAS, granting suitable levels of interoperability for integration in controlled airspace. Some theoretical models underlying key functionalities of the Next Generation Mission Management Systems (NG-FMS) are presented, particularly including 4-Dimensional Trajectory (4DT) prediction and optimisation algorithms currently being implemented [35]. Despite the dense air traffic, controlled airspace can present opportunities for RPAS integration as the expert processing required is considerably lower and this has the potential to reduce the complexity of conflict resolution algorithms, leading to significant economic and safety benefits.

\section{REFERENCES}

[1] NASA, "UTM: Air Traffic Management for Low-Altitude Drones", National Aeronautics and Space Administration (NASA) NASAfacts NF-2015-10-596-HQ, Washington DC, USA, 2015.

[2] P. H. Kopardekar, "Safely enabling UAS operations in low-altitude airspace", NASA UAS Traffic Management (UTM), Moffett Field, CA, USA, 2015.

[3] Amazon Inc., "Determining safe access with a best-equipped, bestserved model for small unmanned aircraft systems (white paper)", Amazon Inc., 2015.

[4] Amazon Inc., "Revising the airspace model for the safe integration of small unmanned aircraft systems (white paper)", Amazon Inc., 2015.

[5] Google Inc., "Google UAS airspace system overview (white paper)", Google Inc., 2015.

[6] B. Stevenson, "NASA plans next phase of UAV ATM integration", Flight Global, vol. 23 October, 2015

[7] D. Gettinger and A. Holland Michel, "Drone sightings and close encountes: an analysis", Center for the Study of the Drone, Bard College, Annandale-on-Hudson, NY, USA, 2015.

[8] B. Carey, "Lockheed Martin: simple ATC mods would allow drone flights", Aviation International News - AINonline, vol. 8th December, 2015

[9] ICAO, "Annex 11 to the Convention on International Civil Aviation Air Traffic Services", International Civil Aviation Organization (ICAO), Montreal, QC, Canada, 2001.

[10] B. T. Clough, "Unmanned Aerial Vehicles: Autonomous Control Challenges, A Researcher's Perspective", Journal of Aerospace Computing, Information, and Communication, vol. 2, pp. 327-347, 2005. DOI: $10.2514 / 1.5588$

[11] ICAO, "Annex 2 to the Convention on International Civil Aviation Rules of the Air", The International Civil Aviation Organization (ICAO), Montreal, Canada, 1990.

[12] B. T. Clough, "Unmanned aerial vehicles: autonomous control challenges, a researcher's perspective", in Cooperative Control and Optimization, ch. 3, pp. 35-53, P. Murphey and P. M. Pardalos, Eds., Kluwer Academic Publishers, Netherlands, 2002.

[13] T. B. Sheridan, Telerobotics, Automation, and Human Supervisory Control, MIT Press, Cambridge, MA, USA, 2003.

[14] ICAO, "Working Document for the Aviation System Block Upgrades - The Framework for Global Harmonization", The International Civil Aviation Organization (ICAO), 2013.

[15] S. Ramasamy and R. Sabatini, "A Unified Approach to Cooperative and Non-Cooperative Sense-and-Avoid", 2015 International Conference on Unmanned Aircraft Systems (ICUAS '15), Denver, CO, USA, 2015. DOI: 10.1109/ICUAS.2015.7152360

[16] S. Ramasamy, A. Gardi, J. Liu, and R. Sabatini, "A Laser Obstacle Detection and Avoidance System for Manned and Unmanned Aircraft Applications", 2015 International Conference on Unmanned Aircraft Systems (ICUAS '15), Denver, CO, USA, 2015. DOI: 10.1109/ICUAS.2015.7152332

[17] FAA, "Integration of Civil Unmanned Aircraft Systems (UAS) in the National Airspace System (NAS) Roadmap", Federal Aviation Administration (FAA), 1st ed., 2013.

[18] L. Bouwmeester, R. Clothier, R. Sabatini, and G. Williams, "Autonomous Communication Between Air Traffic Control and Remotely Piloted Aircraft", 16th Australian International Aerospace Congress (AIAC16), Melbourne, Australia, 2015, pp. 48-57

[19] RMIT News, "Talking drone offers aviation safety boost", 2015. URL: http://www.rmit.com/news/all-news/2015/june/talking-droneoffers-aviation-safety-boost/

[20] ARINC, "Advanced Flight Management Computer System - ARINC Characteristic 702A-3", Airlines Electronic Engineering Committee, Aeronautical Radio Incorporated (ARINC), 2006. 
This is the author pre-publication version. This paper does not include the changes arising from the revision, formatting and publishing process. The final paper that should be used for referencing is:

A. Gardi, S. Ramasamy, R. Sabatini and T.Kistan, "CNS+A Capabilities for the Integration of Unmanned Aircraft in Controlled Airspace." Proceedings of IEEE International Conference on Unmanned Aircraft Systems (ICUAS 2016). Arlington, VA (USA), June 2016.Print ISBN: 978-1-4673-9333-1

[21] R. Sabatini, T. Moore, C. Hill, and S. Ramasamy, "Assessing Avionics-Based GNSS Integrity Augmentation Performance in UAS Mission- and Safety-Critical Tasks", 2015 International Conference on Unmanned Aircraft Systems (ICUAS '15), Denver, CO, USA, 2015

[22] R. Sabatini, T. Moore, and C. Hill, "Avionics-Based GNSS Augmentation for Unmanned Aerial Systems Sense-and-Avoid", 26th International Technical Meeting of the Satellite Division of the Institute of Navigation (ION GNSS+ 2014), Tampa, FL, USA, 2014

[23] R. Sabatini, T. Moore, and C. Hill, "A new avionics-based GNSS integrity augmentation system: Part 1 - Fundamentals", Journal of

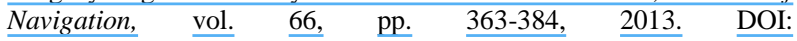
$10.1017 / \mathrm{S} 03734 \overline{63313000027}$

[24] R. Sabatini, T. Moore, and C. Hill, "A new avionics-based GNSS integrity augmentation system: Part 2 - Integrity flags", Journal of Navigation, $\quad$ vol. $\quad 66, \quad$ pp. $\quad 501-522, \quad$ 2013. $\quad$ DOI: 10.1017/S0373463313000143

[25] R. Sabatini, A. Gardi, S. Ramasamy, T. Kistan, and M. Marino, "Modern Avionics and ATM Systems for Green Operations", in Encyclopedia of Aerospace Engineering, ch. eae1064, R. Blockley and W. Shyy, Eds., John Wiley \& Sons, 2015.

[26] A. Gardi, R. Sabatini, and S. Ramasamy, "Multi-objective optimisation of aircraft flight trajectories in the ATM and avionics context", Progress in Aerospace Sciences, vol. 83, 2016. DOI: 10.1016/j.paerosci.2015.11.006

[27] A. Gardi, S. Ramasamy, and R. Sabatini, "4-Dimensional Trajectory Generation Algorithms for RPAS Mission Management Systems", 2015 International Conference on Unmanned Aircraft Systems (ICUAS '15), Denver, CO, USA, 2015. DOI: 10.1109/ICUAS.2015.7152314

[28] S. Ramasamy, R. Sabatini, and A. Gardi, "Novel Flight Management Systems for Improved Safety and Sustainability in the CNS+A Context", IEEE/AIAA Integrated Communication, Navigation and Surveillance Conference (ICNS 2015), Herndon, VA, USA, 2015. DOI: 10.1109/ICNSURV.2015.7121225
[29] A. V. Rao, "Survey of Numerical Methods for Optimal Control", Advances in the Astronautical Sciences, vol. 135, pp. 497-528, 2010

[30] RTCA, "Minimum Aviation System Performance Standards: Required Navigation Performance for Area Navigation", Radio Technical Committee for Aeronautics Inc. (RTCA) RTCA DO-236C Washington DC, USA, 2013.

[31] RTCA, "Minimum Operational Performance Standards for Global Positioning System/Satellite-Based Augmentation System Airborne Equipment", Radio Technical Commission for Aeronautics Inc. (RTCA) RTCA DO-229D, Change 1 ed., Washington DC, USA, 2013.

[32] A. Gardi, R. Sabatini, S. Ramasamy, and T. Kistan, "Real-Time Trajectory Optimisation Models for Next Generation Air Traffic Management Systems", Applied Mechanics and Materials, vol. 629, pp. 327-332, 2014. DOI: 10.4028/www.scientific.net/AMM.629.327

[33] I. A. B. Wilson, "Trajectory Negotiation in a Multi-sector Environment", Programme for Harmonised Air Traffic Management Research in EUROCONTROL (PHARE), Bruxelles, Belgium, 1998.

[34] A. Gardi, R. Sabatini, S. Ramasamy, and K. de Ridder, "4Dimensional Trajectory Negotiation and Validation System for the Next Generation Air Traffic Management", AIAA Guidance, Navigation, and Control Conference (GNC 2013), Boston, MA, USA, 2013. DOI: $10.2514 / 6.2013-4893$

[35] A. Gardi, R. Sabatini, T. Kistan, Y. Lim, and S. Ramasamy, "4Dimensional Trajectory Functionalities for Air Traffic Management Systems", IEEE/AIAA Integrated Communication, Navigation and Surveillance Conference (ICNS 2015), Herndon, VA, USA, 2015. DOI: 10.1109/ICNSURV.2015.7121246

[36] S. Ramasamy, R. Sabatini, A. Gardi, and T. Kistan, "Next Generation Flight Management System for Real-Time Trajectory Based Operations", Applied Mechanics and Materials, vol. 629, pp. 344-349, 2014. DOI: 10.4028/www.scientific.net/AMM.629.344 